\title{
Adaptation effects on evoked electrical taste
}

\author{
Z. BUJAS, SILVIJA SZABO, MIRJANA KOVAČIĆ, and A. ROHAČEK \\ University of Zagreb, Dure Salaja br. 3, 41000 Zagreb, Yugoslavia
}

\begin{abstract}
In spite of a clear increment in absolute thresholds as an effect of self-adaptation, the electrically provoked taste sensation does not show a reduction in subjective intensity during the protracted stimulation time. Chemical cross-adaptation effects on the threshold and suprathreshold levels of electrical taste are observed only when the adapting solutions are electrolytes regardless of their taste qualities. It seems that the stability of perceived intensity during current flow is principally due to some irritation of the sensitive tissue of the tongue, whereas cross-adaptation effects may be attributed to the potential changes across the excitable membranes caused by electrical properties of adapting electrolytic solutions.
\end{abstract}

In one of the first studies concerning electrical taste, Volta (1792) observed that the electrically provoked taste sensation lasted all the time during the current flow and that in the course of that period taste intensity went up.

In opposition to the well-known gustatory adaptation phenomena, does this statement indicate that the sensory structures that are the site of adaptation changes are bypassed in the electrical stimulation of the tongue?

The purpose of this investigation was to check the correctness of Volta's observation and to collect some data on the influence of the protracted stimulus action time and on cross-adaptation effects on evoked electrical taste.

\section{GENERAL METHOD}

Electrical taste was evoked by means of anodal or cathodal direct current. An electrical stimulator and nonpolarizable $\mathrm{Ag}: \mathrm{AgCl}$ electrodes dipped in $0.1 \% \mathrm{NaCl}$ were used. The stimulation was monopolar. The contact between the active electrode and the tongue was made by a plug of agar with a surface of about $60 \mathrm{~mm}^{2}$. The indifferent electrode was a bath for fingers of the left hand with a contact surface of about $50 \mathrm{~cm}^{2}$. The electrodes were in series with a resistance of $30 \mathrm{Kohms}$ or greater. To avoid transients at the onset of direct current stimulation, a special device was used to obtain a rapid but smooth onset of the stimulus.

In the experiments with cross adaptation, the taste solutions were applied by means of a flow system through a glass stimulator. The solution flowed over a limited surface of the tongue $\left(1.8 \mathrm{~cm}^{2}\right)$ with a speed of $15 \mathrm{~cm}^{3} / \mathrm{sec}$.

Four trained Os took part in the experiments.

\section{EXPERIMENT I: \\ PERCEIVED INTENSITY OF ELECTRICAL TASTE BEFORE, AT THE END OF, AND AFTER ADAPTATION}

The Os were instructed to estimate the overall subjective intensity of electrical stimuli. The perceived intensity was evaluated by the Stevens (1969) procedure of direct magnitude estimation, with the standard set equal to 10 . The standard was given before each stimulus was to be evaluated. In experiments with the anode, the standard was 70 micro $A$ and the stimulation lasted $3 \mathrm{sec}$. For cathodal stimulation the standard was 200 microA and the duration was $4 \mathrm{sec}$.

Five adapting current intensities were applied in each of the two kinds of electrical stimulation. The adaptation time was $20 \mathrm{sec}$. Three seconds before the end of the adaptation time a signal was given, and the task of the $\mathbf{O}$ was to consider the subjective intensity of taste in only these last $3 \mathrm{sec}$ of current flow. At the end of $20 \mathrm{sec}$ the electrode was removed, and then, after the estimate was given, the $S$ replaced the electrode on the same part of the tongue and received a test stimulus of the same objective intensity as that of the preceding adapting stimulus. The test stimulus lasted $3 \mathrm{sec}$ for the anode and $4 \mathrm{sec}$ for the cathode. The subjective intensity of this second stimulus was estimated too. Between the end of adaptation and this second stimulus, about $10 \mathrm{sec}$ elapsed. The adapting stimuli were applied in random order.

In the control measurements, the $\mathrm{O}$ held the active electrode in contact with the tongue for $17 \mathrm{sec}$ without any current flow and then was stimulated with different test intensities.

In that manner we obtained three kinds of magnitude estimates for the same current intensities in relation to the fixed standard: one without previous adaptation, one at the end of the adaptation time, and one $10 \mathrm{sec}$ after the end of adaptation. Twelve sessions were run with each 0,6 for anodal and 6 for cathodal stimulation. Each result is the median based on at least 48 evaluations. The results are shown in Fig. 1.

From these results it seems that Volta's observation was correct at least in the sense that the perceived overall intensity of electrical stimulus shows no reduction during the protracted stimulus action time. However, the psychophysical functions obtained in the recovery period clearly indicate that the previous adapting stimulus had a depressing influence on the response of the involved sensory structures. In all Os, after adaptation, the test stimuli evoked a less intense overall sensation.

The results obtained may be due to several factors. 


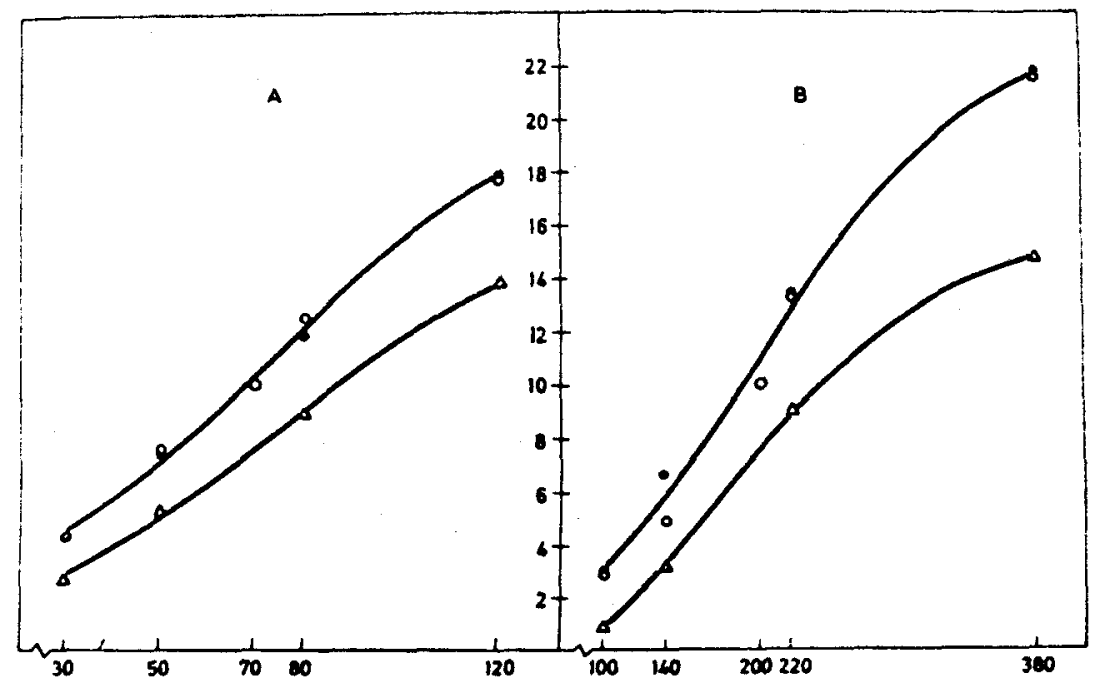

Fig. 1. Psychophysical functions for anodal (A) and cathodal (B) taste under nonadaptation and adaptation conditions. Open circles: median magnitude estimates without previous adaptation; filled circles: estimates at the end of adaptation period; triangles, estimates $10 \mathrm{sec}$ after the end of adaptation.

First, it is possible that the stimulus intensity during a constant current flow is not steady but grows with time. In this case, some accumulation of electrolytic products may compensate the influence of adaptation. Second, after a certain time, the current may irritate nongustatory sensitive tissues of the tongue, thus influencing the subjective overall intensity. Third, during adaptation, the differential sensitivity may grow and so compensate the increase in the absolute threshold.

The possibility of a progressive accumulation of electrolytic products as a cause of the relative stability of electrical taste intensity during the current flow seems to be less important than the irritation of the sensitive tissue of the tongue. This is at least partially supported by electrophysiological data on the neural activity in rats' chorda tympani. The integrated nerve activity evoked by a continuous anodal stimulation of the rat tongue shows, after an initial peak, a rapid decrement followed by a slow decrease very similar to changes in the nerve activity obtained during a continuous flow of a taste solution (see Fig. 2).
It is possible to object that the estimates made in the last $3 \mathrm{sec}$ of the adapting period might have been biased. The reduction of taste intensity might exist but not be signaled by the $O$ because it progresses too slowly to be easily detected. Besides, owing to the influence of the "adaptation level" in the sense of Helson's theory (1947), the procedure of direct magnitude estimation may not have been appropriate for allowing the observation of the continuous adaptation effect. To check these possibilities, an additional experiment was made in which the Os equated the subjective intensity of the sensation at the end of adaptation with the subjective effect of a short test stimulus applied to another nonadapted part of the tongue. To determine subjective equality, the constant method was used, with a counterbalanced procedure to neutralize the space error. Subjective equality was determined for only one medium intensity of anodal and cathodal current.

The results, based on 60 series of measurements for each 0 , are in agreement with those obtained with the Stevens procedure. At the end of the adaptation time

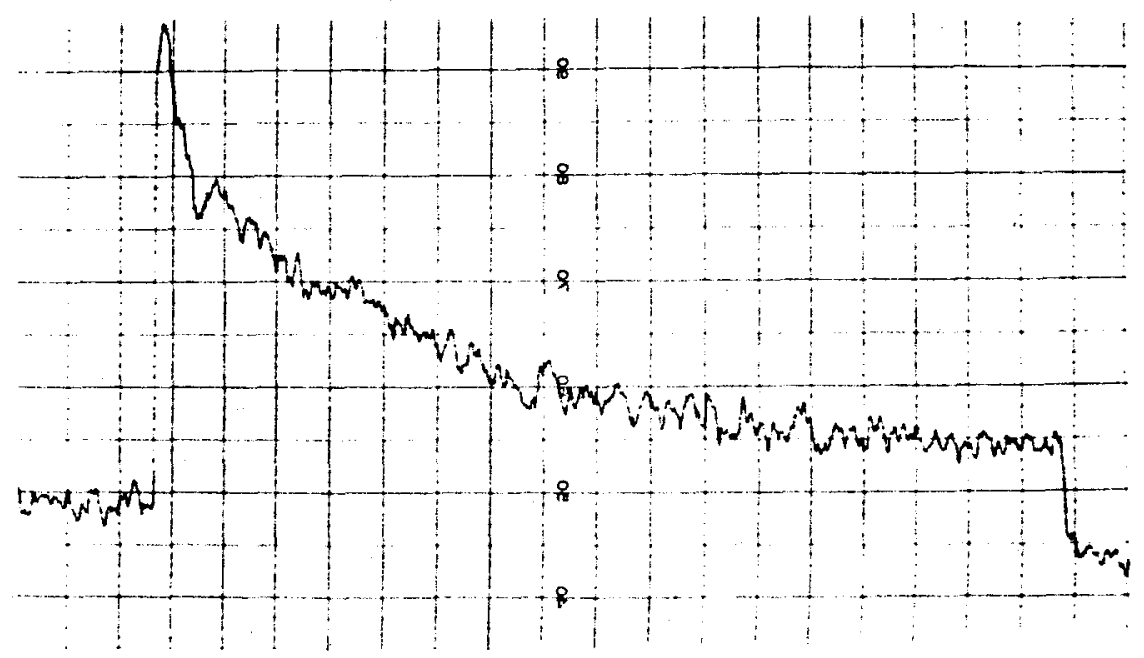

Fig. 2. Decrement of integrated activity of rat's chorda tympani during continuous anodal stimulation (after Frank, Pfaffmann, \& Bujas, unpublished data). 
Fig. 3. Influence of partial adaptation to different matched solutions on the threshold for anodal and cathodal taste. On the ordinate: threshold values after cross adaptation expressed in proportion to the threshold after the water flow $(=1)$. On the abscissa: the adapting substances ranked according to their influence on the anodal threshold. $\mathbf{N}=$ sodium chloride, $\mathbf{A}=$ citric acid, $\mathbf{Q}=$ quinine hydrochloride, $\mathbf{S}_{\mathbf{c}}=$ sodium cyclamate, $\mathrm{S}=$ sucrose, $\mathrm{C}=$ caffeine. Asterisks mark the differences significant at the $p<.01$ or $p<.05$ level.

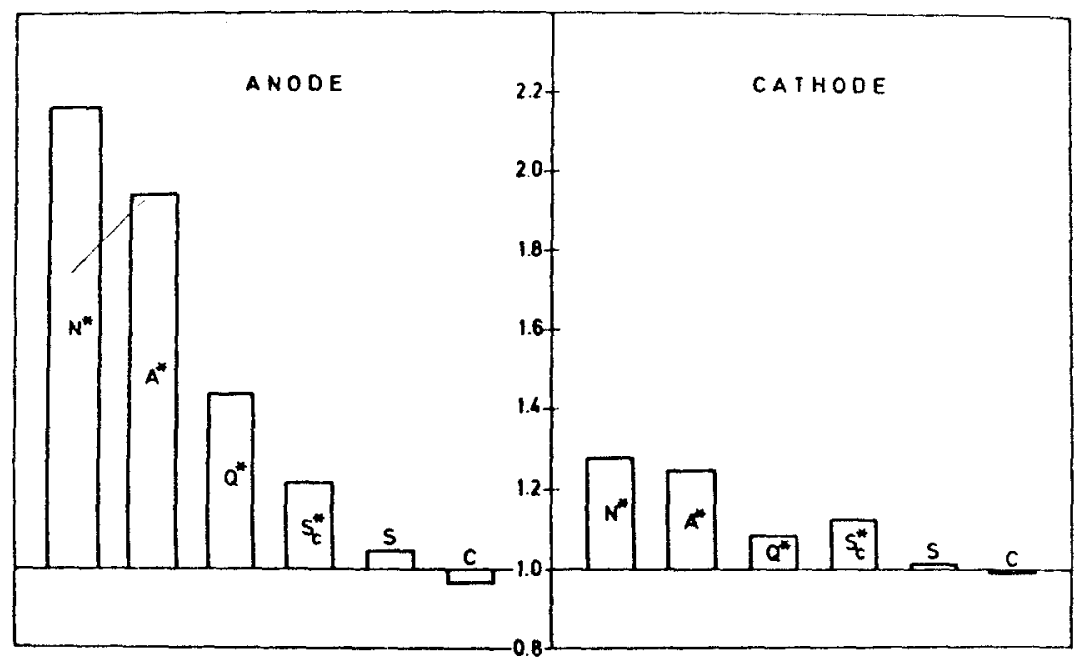

nonadaptation conditions and after adaptation to different taste solutions were determined by the constant method. The order of measurements was randomized. For cross adaptation, six substances were used. The concentrations of adapting solutions were approximately equated in overall subjective intensity to 1.0M NaCl. The medians of the matched solutions were: $1.0 \mathrm{M} \mathrm{NaCl}, 0.064 \mathrm{M}$ citric acid, $0.5 \mathrm{M}$ sucrose, $0.06 \mathrm{M}$ sodium cyclamate, $0.0096 \mathrm{M}$ quinine hydrochloride, and $0.2 \mathrm{M}$ caffeine. The solutions and water were kept at $35^{\circ} \mathrm{C}$.

In the control experiments, water, instead of solutions, flowed over the tongue. The adapting solutions or water flowed for $20 \mathrm{sec}$. To avoid the influence of residual traces of the solutions, after adaptation the tongue was rinsed with water for about $4 \mathrm{sec}$. Between the end of adaptation (or of the water flow) and the electrical test stimulus, about $10 \mathrm{sec}$ elapsed. The electrical test stimulus was applied for 3 and $4 \mathrm{sec}$, respectively.

The threshold values of each $O$ are the mean of at least 20 series of measurements. The results are presented in Fig. 3. The adaptation effects are expressed in proportion to the normal threshold value (after the water flow) obtained in the same session.

As Fig. 3 shows, the degree of the cross-adaptation effect, measured by threshold changes, differs according to the substance and the stimulation modality applied. In general, the influence of cross adaptation is more pronounced for anodal than for cathodal thresholds. The adaptation to sodium chloride and citric acid led to the most significant increase, with lesser effects of quinine and sodium cyclamate, while sucrose and caffeine had no statistically significant effects. The results presented in Fig. 3 are the mean values. However, the individual data also show the same rank order in spite of some differences in solutions which were subjectively equal.

As the obvious dominant taste quality evoked by anodal current is sour, it may be surprising that the increment of the anodal threshold after $\mathrm{NaCl}$ adaptation

The thresholds for electrical tastes both in 
is the same or even higher than the increment after citric acid adaptation. Similarly, the cathodal taste, which is a mixture of bitter and sweet, is more influenced by $\mathrm{NaCl}$ and citric acid than by sweet or bitter solutions.

On the other hand, sweet and bitter substances increase not only the cathodal but also the anodal threshold if the solutions are electrolytes.

These cross-adaptation effects can hardly be interpreted as a sign of some commonality of taste receptors activated by electrolytic solutions on the one hand and by electric current on the other. Against the assumption of the existence of a type of taste receptor generally sensitive to electrolytes speaks the fact that between the electrolytes of different taste qualities there is no generalization of adaptation effects. It is therefore more probable that, in our stimulation conditions, the efficacy of electrical stimulus was to some extent reduced by potential changes across the excitable membranes caused by electrical properties of adapting electrolytic solutions. Further, some independence of the electrical taste thresholds from the taste qualities of adapting solutions suggests that, at least partially, the first step in chemical stimulation is bypassed in electrical stimulation.

The results obtained in an additional experiment with electrical cross adaptation showed that after adaptation to anodal current of 60 microA during $20 \mathrm{sec}$, the threshold for the onset of cathodal current was increased by about $18 \%$, and after adaptation to cathodal current of 200 microA, the anodal threshold was about $16 \%$ higher than in nonadaptation conditions. These differences, at the lower limit of significance, are too small to allow the assumption of some commonality of the receptor mechanism or of a similarity in factors influencing anodal and cathodal taste.

The adaptation effect of the same kind of current on the corresponding taste threshold (self-adaptation) was obviously higher: the increase amounted to about $56 \%$ for the cathode and $85 \%$ for the anode.

\section{EXPERIMENT III: CROSS-ADAPTATION EFFECTS ON THE SUPRATHRESHOLD LEVEL}

Electrical tastes, especially the taste provoked by cathodal current, are not monogustatory. Thus, it was of interest to check possible changes in the taste spectra in addition to the influence of adaptation on the subjective overall intensity. Using a similar procedure to that of Smith and McBurney (1969), we asked our Os to rate the overall subjective intensity of a short suprathreshold stimulus and then to divide this estimate among the evoked taste qualities. The same stimuli were rated once after the water flow and once under adaptation to the six solutions already mentioned. The current intensity was 60 microA for the anodal test stimulus and 200 microA for the cathodal test stimulus; these intensities were approximately of equal subjective intensity. The measurements with the solutions were completed with the data obtained with the current as adapting stimulus.

In evaluating the overall intensity, the perceived intensity of the electrical test stimulus after the water flow or the blank contact was taken as the modulus $(=100)$.

From a very large number of evaluations, only few statistically significant differences were obtained. Neither the perceived overall intensity of anodal stimulation nor that of cathodal stimulation was significantly influenced by adaptation to quinine, caffeine, sodium cyclamate, or sucrose. It is interesting that adaptation to quinine and sodium cyclamate which increased the anodal and, to some extent, also the cathodal threshold did not influence the suprathreshold level.

Among the solutions, only adaptation to sodium chloride significantly decreased the overall subjective intensity for both kinds of electrical stimulation. However, the degree of the influence is different from that regarding the threshold level. While after adaptation to $\mathrm{NaCl}$ the threshold increase was higher for the anodal (116\%) than for cathodal stimulation (28\%), the decrement in total subjective intensity was $12 \%$ for the anode and $33 \%$ for the cathode.

Apparently the largest divergence in the influence of cross adaptation is that relating to citric acid. The adaptation to citric acid reduces the overall intensity of anodal stimulus (8\%) but increases the perceived intensity of cathodal stimulus (11\%). This potentiation for cathodal stimulus is probably due to some irritation of the sensitive tissue caused by citric acid. The relatively strong cathodal current could in these circumstances stimulate also tactile and pain receptors which may contribute to overall intensity.

Finally, the reduction in perceived overall intensity is statistically significant also after cross adaptation with the current. After adaptation to 200 microA of cathodal current, the anodal overall intensity is reduced by about $12 \%$, and after adaptation to 60 micro $A$ of anodal current the cathodal overall intensity is reduced by about $7 \%$.

In the case of electrical taste profiles, small differences between adaptation and nonadaptation conditions were observed. For the anodal taste a small decrement in overall intensity (after $\mathrm{NaCl}$, citric acid, and cathodal current) is related to a reduction of sourness, but the anodal taste after adaptation is almost monogustatory as it was before. On the other hand, the composite cathodal sweet-bitter taste shows irregular changes after adaptation to $\mathrm{NaCl}$, citric acid, and anodal current, stimuli which provoke a change in overall cathodal taste intensity. After adaptation, the bitter taste is sometimes reduced and the "undefined" taste increased (as after citric acid). Sometimes the sweet taste is increased (as after anodal current), but these changes were not reported by all Os. It is interesting that the degree of 


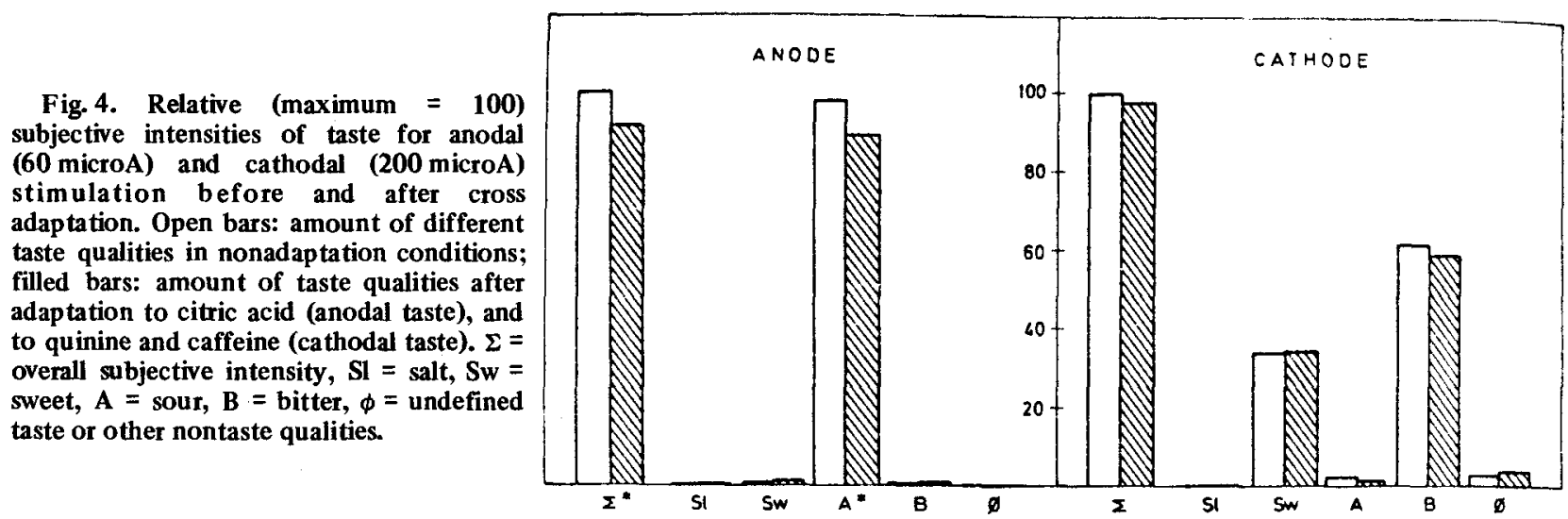

sweetness or bitterness in the composite cathodal suprathreshold taste was not significantly affected by adaptation to sweet or bitter solutions.

As an example, Fig. 4 shows the electrical taste profiles before and after adaptation to the solutions which have a taste quality similar to that provoked by electric current of a given polarity. The normal anodal perceived intensities are compared with those obtained after adaptation to citric acid, while the cathodal perceived intensities are compared with the average ones obtained after adaptation to two bitter substances. The sourness of the anodal taste is somewhat reduced, but the same and an even more pronounced effect are produced by the adaptation to $\mathrm{NaCl}$ and cathodal current, which are not sour. On the other hand, the bitterness of the cathodal taste shows no significant change after adaptation to two bitter solutions.

\section{CONCLUSION}

The relative stability of overall electrical taste intensity during a protracted stimulus action time does not mean an absence of adaptation. This stability is presumably due to some irritation of the sensitive tissue of the tongue and also, to a lesser extent, to some progressive increase of electrolytic products during the current flow. Adaptation in the form of a decrement of suprathreshold response exists, but this adaptation effect can be established only after the cessation of the adapting stimulus when the irritation diminishes and the electrolytic products dissipate.

The adaptation as the threshold increases for the same stimulus is similar to that observed in chemical gustatory adaptation. Thus, if electrical stimulation bypasses the first step in chemical stimulation, that first step is not the only site where the adaptation changes can occur.

Cross adaptation as an approach to check whether or not the receptors activated by two stimuli are common has given no clear response. The adapting solutions, regardless of their taste qualities, increase the threshold for electrical stimuli provided the solutions are electrolytes. These results, if confirmed by further data, suggest that electrolytic solutions change the resting potential of taste excitable structures, which has an influence on the efficacy of electrical stimulation.

The effects of cross adaptation are to some extent different at threshold and above threshold. Some electrolytic solutions that to a significant degree increase the thresholds have no effect on the suprathreshold level. This is one more indication that the adaptation effects on threshold and suprathreshold levels ought not be the same.

The psychophysical functions of electrical taste sensation have a sigmoid form and are similar to certain magnitude functions found by Stevens (1969) in the case of taste elicited by chemical stimulation.

The fact that adaptation to some electrolytes, such as $\mathrm{NaCl}$ and citric acid, has an influence on the threshold and suprathreshold levels of electrical taste regardless of the dissimilarity in their taste quality, and yet that some solutions of a taste quality similar to that of the electrical taste produce no effect suggests the existence of some differences between the receptor mechanisms of chemical and electrical taste. It seems that electrical taste is due in part to the chemoelectrolytic action on the cell membrane and in part to the electrolysis of the intracellular fluid and a direct action on the nerve fibers.

\section{REFERENCES}

Helson, H. Adaptation-level as frame of reference for prediction of psychophysical data. American Journal of Psychology, $1947,60,1-29$.

Smith, D. V., \& McBurney, D. H. Gustatory cross-adaptation: Does a single mechanism code the salty taste? Journal of Experimental Psychology, 1969, 80, 101-105.

Stevens, S. S. Sensory scales of taste intensity. Perception \& Psychophysics, 1969, 6, 302-308.

Volta, A. (1792) Briefe über thierische Electricität. In A. J. Oettingen (Ed.), Ostwald's Klassiker der exakten Wissenschaften. Leipzig: Englemann, 1900.

(Received for publication March 19, 1973; revision received July 21,1973 ; second revision received September 17, 1973.) 